\title{
Whole exome sequencing identifies a novel missense FBN2 mutation co-segregating in a four-generation Chinese family with congenital contractural arachnodactyly
}

Xingping Guo ${ }^{1}$, Chunying Song ${ }^{1 *}$, Yaping Shi ${ }^{1}$, Hongxia Li ${ }^{1}$, Weijing Meng ${ }^{1}$, Qinzhao Yuan ${ }^{1}$, Jinjie Xue ${ }^{1}$, Jun Xie ${ }^{1}$, Yunxia Liang ${ }^{1}$, Yanan Yuan' ${ }^{1}$, Baofeng Yu ${ }^{1}$, Huaixiu Wang ${ }^{1}$, Yun Chen ${ }^{1}$, Lixin Qi ${ }^{1}$ and Xinmin $\mathrm{Li}^{2^{*}}$

\begin{abstract}
Background: Congenital contractural arachnodactyly (CCA) is an autosomal dominant rare genetic disease, estimated to be less than 1 in 10,000 worldwide. People with this condition often have permanently bent joints (contractures), like bent fingers and toes (camptodactyly).

Case presentation: In this study, we investigated the genetic aetiology of CCA in a four-generation Chinese family. The blood samples were collected from 22 living members of the family in the Yangquan County, Shanxi Province, China. Of those, eight individuals across 3 generations have CCA. Whole exome sequencing (WES) identified a missense mutation involving a T-to-G transition at position 3229 (c.3229 T > G) in exon 25 of the FBN2 gene, resulting in a Cys 1077 to Gly change (p.C1077G). This previously unreported mutation was found in all 8 affected individuals, but absent in 14 unaffected family members. SIFT/PolyPhen prediction and protein conservation analysis suggest that this novel mutation is pathogenic. Our study extended causative mutation spectrum of FBN2 gene in CCA patients.
\end{abstract}

Conclusions: This study has identified a novel missense mutation in FBN2 gene (p.C1077G) resulting in CCA in a family of China.

Keywords: Whole exome sequencing, FBN2, Mutation, Congenital contractural arachnodactyly

\section{Background}

CCA, also known as Beals-Hecht syndrome, is an autosomal dominant disorder of connective tissue. It was first described by Epstein et al. in 1968, and then was differentiated from Marfan syndrome (MFS) by Beals and Hecht in 1971 [1-3]. MFS and CCA share numerous common characteristics, such as a so-called marfanoid appearance constituted by tall, slender, asthenic appearance and skeletal features such as arachnodactyly, dolichostenomelia, pectus deformities, and kyphoscoliosis. In contrast with MFS, most individuals with CCA have

\footnotetext{
* Correspondence: 16398656@qq.com; xinminli@mednet.ucla.edu

'Shanxi Key Laboratory of Birth Defects and Cell Regeneration, Shanxi Population and Family Planning Research Institute, 11 Beiyuan Street, Taiyuan, Shanxi 030006, People's Republic of China

${ }^{2}$ Department of Pathology and Laboratory Medicine, UCLA, Los Angeles, CA 90095, USA
}

"crumpled" ears, flexion contractures, and muscular hypoplasia. fex mictictic in elastic providing strength and joints and connective tissue that supports the body's de tion of a fibillion $F B N 2$ is expressed early in embryogenesis in elastic cartilage, aortic tunica media and bronchial epithelium [7]. FBN2 deficient mice have forelimbs contractures, bilateral syndactyly and disorganized microfibrils [8].

As of September 5, 2016, 63 FBN2 mutations have been described in 72 probands/families with CCA [6]. 
Because $F B N 2$, located at $5 \mathrm{q} 23.3$, is a very large gene containing 65 exons and encoding 2912 amino acids, we thought many more mutations causative to CCA have yet to be identified. Here, we report a novel missense mutation in FBN2 gene identified by whole exome sequencing in a four-generation CCA family.

\section{Case presentation}

The subjects of the study were 22 living members from a four-generation family affected with CCA living in the Yangquan County, Shanxi Province, China. The study was reviewed and approved by the institutional ethics committee of the Shanxi Population and Family Planning Research Institute in Shanxi, China. A written informed consent was obtained from the subjects before study. $7 \mathrm{ml}$ peripheral venous blood was collected from 8 CCA patients (II-3, II-5, II-7 III-3, III-4, III-5. III-7, IV-1) and 15 normal subjects (II-1, II-2, II-4. II-6. II-8, II-9, II-10, II-11, II-12, III-1, III-2, III-6, III-8, III-9, III-10) in a Lavendar EDTA tube. The proband was the grandson III-5 (Fig. 1).

\section{Materials and methods DNA extraction}

Genomic DNA was extracted from the blood samples using a DNA isolation kit for mammalian blood (Tiangen Biotech, China) according to manufacturer's instructions. The integrity of DNA was evaluated using an Agilent 2100 Bioanalyzer (Agilent Technologies, Palo Alto, CA, USA) and purity/concentration was determined using a NanoDrop 8000 Spectrophotometer (Thermo Scientific, Wilmington, DE, USA).

\section{Sequencing and analysis}

Whole exome sequencing was performed using Roche NimbleGen SeqCap EZ V3.0 System according to the manufacturer's instruction. Briefly, 1ug of genomic DNA was sheared, size selected to roughly 300 bps. DNA library was made using Kapa LTP library preparation kit, followed by incubation with SeqCap biotinylated DNA baits. After an amplification of 14 cycles, the libraries were sequenced at $2 \mathrm{X} 100$ on a HiSeq 2500 sequencer.

The raw sequence data were aligned to the GRCh37 human reference genome using BWA v0.7.7-r411. PCR duplicates were marked using Mark Duplicates program in Picard-tools-1.115 tool set. GATK v3.2-2 and Samtools were used for the identification of INDEL and the SNVs, respectively. All variants were annotated using the Annovar program. FBAT (family based associated test) was used to identify SNPs that are significantly associated with CCA at the $p$ value of 0.05 .

\section{Results}

Clinical features of the patients

The studied family has 25 members across four generations. Three of those were deceased (Fig. 1). A detailed physical examination was taken for 22 living members when samples were collected. Eight living members of the family have a typical CCA phenotype (II-3, II-5, II-7, III-3, III-4, III-5, III-7, IV-1) as presented with slender, contractural clubbed fingers and toes (Fig. 2). III-5 was the proband of 28 years old (at birth with clenched position of hands and 300 of myopia). No neurological, cardiovascular abnormalities, external ear malformation or eye abnormality were noted. Intrafamilial variation in phenotypic expression are modest.

\section{Mutation analysis}

To reconcile clinical findings with molecular data in this family, whole exome sequencing was performed for all 22 living members with an Illumina Hiseq2500 sequencer. All samples were sequenced to a mean target coverage of $>50 x$, and had a quality score of $>$ Q30 for more than $85 \%$ of bases. After identification of all variant calls, FBAT analysis was used to identify variants that were significantly associated with CCA phenotype at $\mathrm{p}<0.05$.

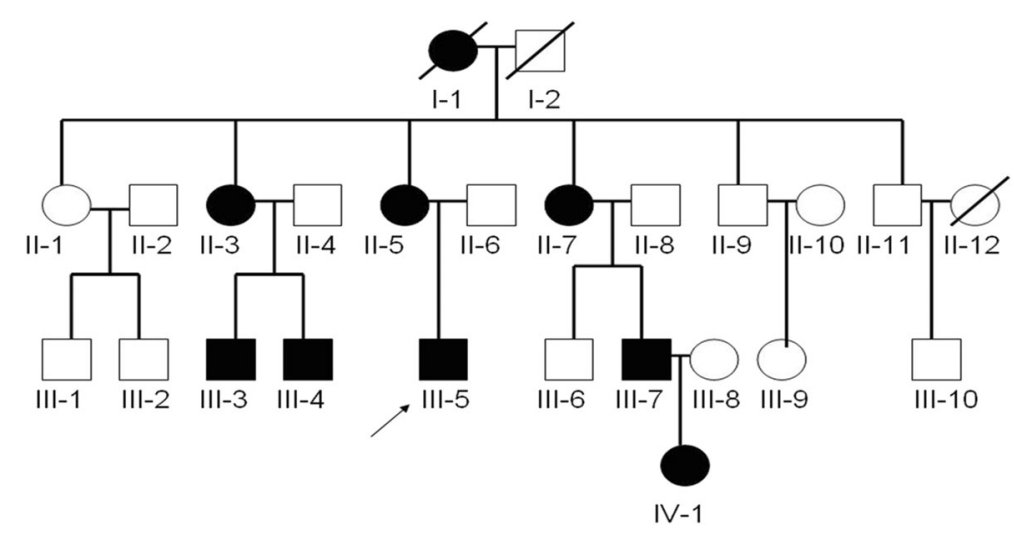

Fig. 1 Pedigree of the family that took part in this study. The patients with congenital contractural arachnodactyly are depicted by black symbols. The arrow indicates the proband. The squares denote males and circles denote females. Crossed lines indicate deceased family members 


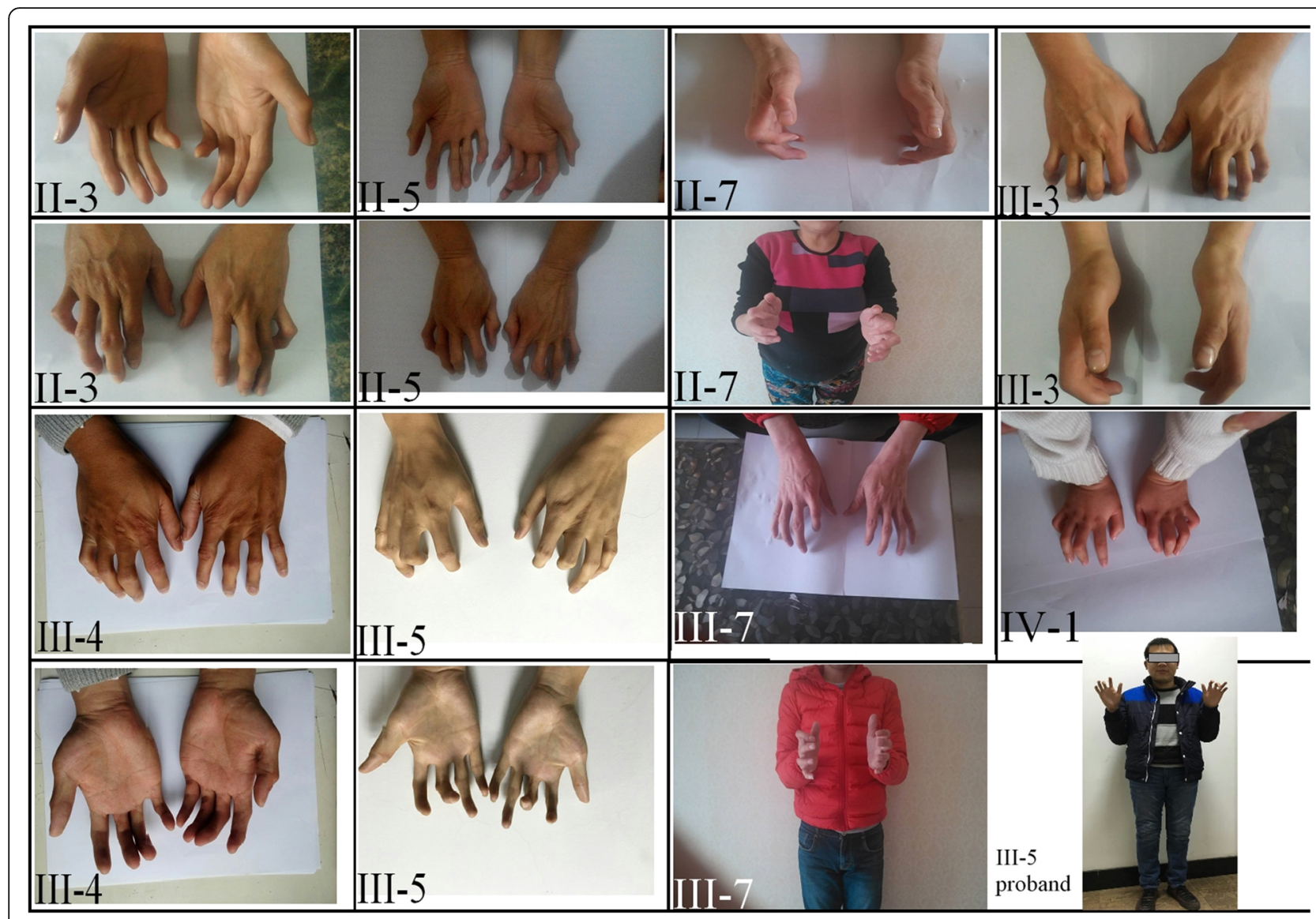

Fig. 2 CCA phenotypes of eight affected family members. The photos of clubbed fingers from II-3, II-5, II-7, III-3; III-4, III-5, III-7 and IV-1 were taken when the samples were collected

Of those variants, heterozygous mutation, T3229G (chr5_127680191, p.C1077G) in FBN2 gene, was the most significant one. This novel missense mutation was $100 \%$ co-segregated with the disease in the family and was absent in 14 unaffected members. C1077G mutation has a CADD PHRED score of 23.4. All six functional prediction tools (SIFT, PolyPhen-2, Mutation Taster, Mutation Assessor, FATHMM and FATHMM MKL Coding) predicted this mutation as damaging. Consistently, amino acid conservation analysis (http://geno me.ucsc.edu) showed that the cysteine at position 1077 (p.C1077) is highly conserved across multiple species
(Fig. 3). This mutation is also confirmed by Sanger sequencing (Fig. 4).

This variant was neither reported in global scale initiatives for variant annotation such as the Exome Aggregation Consortium (ExAC) nor described in the UMD-FBN2 database. However, one mutation located at the same amino acid position (p.Cys1077Arg) was documented in UMDFBN2 database (reported as FRA01BOU F0061 I0092). Interestingly, the phenotype of this patient was also limited to joint contractures but with crumpled ears.

We also found a missense mutation in FNIP1 gene, involving a A-to-G transition at position 2446 (p.I816V) in

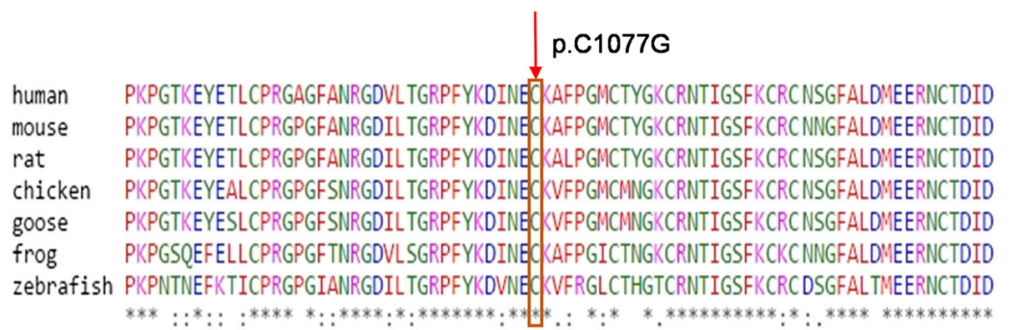

Fig. 3 Protein sequencing alignment showing conservation of cysteine residue at the position 1077 in FBN2 gene 


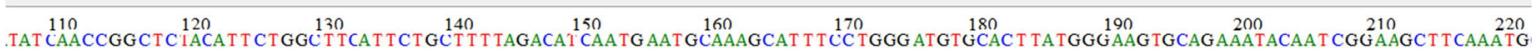
II-3 $\downarrow$

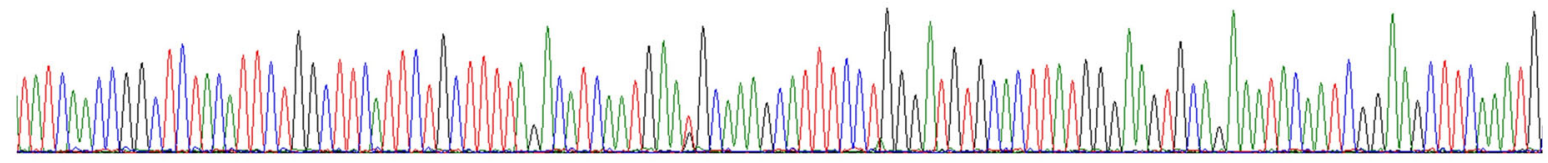

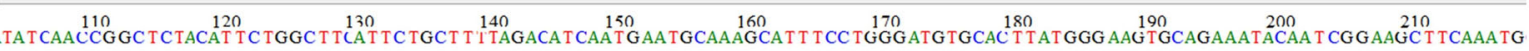

\section{II-1 1}

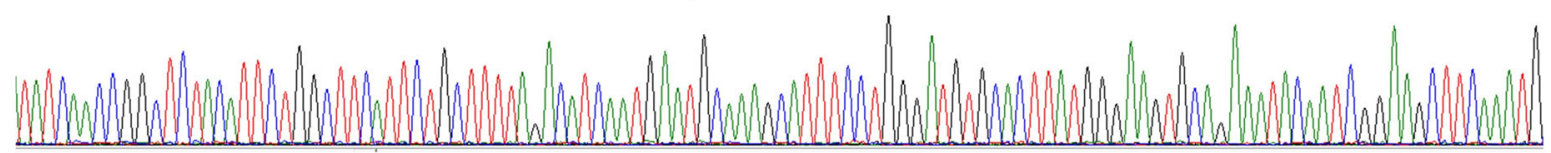

Fig. 4 Sanger sequencing confirmation for the Mutation T3229Gin FBN2 Gene

exon13, which was also $100 \%$ present in CAA patients and absent in all normal members in this family.

\section{Discussion}

CCA is an established autosomal dominant genetic disease [9] characterized by contractures, arachnodactyly, dolichostenomelia, scoliosis, crumpled ears and pectus deformities [10]. The clinical symptoms are similar, but genetically distinct, to MFS. Mutations of FBN2 gene cause CCAand mutations in the FBN1 gene result in MFS. However, MFS is genetically heterogeneous as it can be related to mutations in FBN1, TGFBR2 and TGFBR1 genes [11].

$F B N 2$ gene is located in chromosome $5 \mathrm{q} 23-\mathrm{q} 31$ and contains 65 exons, coding fibrillin-2 (2912 amino acids) [6]. For such a large gene, there are relatively small number of mutations that have been identified so far. As of May 20, 2016, ClinVar only recorded 336 mutations, of which 51 were considered as pathogenic by HGMD Professional database. Fifty-four percent of those pathogenic mutations are missense mutations, frequently replacing the Cysteine with another amino acid. The substitution of another amino acid for Cysteine can alter the structure or function of fibrillin-2 [12, 13]. All of these mutations reduce the amount of fibrillin-2 available to form microfibrils. Decreased microfibril formation will weaken the elasticity of fibers, which leads to the symptoms of CCA [14].

In this study, we found a novel missense mutation involving a T-to-G transition at position 3229 (c.3229 $\mathrm{T}>\mathrm{G}$ ) in exon 25 of $F B N 2$, resulting in a Cys 1077 to Gly change (p.C1077G). Cys 1077 is highly conserved across many different species, suggesting it is required for the function of the protein. As expected, this mutation was predicted to be deleterious by SIFT and PolyPhne- 2 software. Thus, this novel mutation is likely the genetic cause of CCA in this family.

Another missense mutation (rs7717874) in FNIP1 gene, $100 \%$ co-segregated with CCA, has so far not been associated with any disease. It was predicted to be benign with a score of 0.002 (sensitivity: 0.99; specificity: 0.30 ) by PolyPhen-2. FBN2 and FNIP1 are only $310 \mathrm{~kb}$ apart, and thus the association between CCA and FNIP1 mutation is likely due to the linkage disequilibrium. However, we cannot rule out a possibility that FNIP1 gene has a regulatory role in the clinical expression of CCA.

\section{Conclusion}

This study has identified a novel missense mutation in FBN2 gene (p.C1077G) resulting in CCA in a family of China. The role of missense mutation at position 2446 (p.I816V) in FNIP1 gene is worthy of further investigation.

\section{Abbreviations}

CCA: Congenital contractural arachnodactyly; Cly: Clycin; Cys: Cysteine; DNA: Deoxyribonucleic acid; FBAT: Family-based association test; FBN2: Fibrillin-2; FNIP1: Folliculin-interacting protein 1; HGMD: The human gene mutation Database; MFS: Marfan syndrome; PCR: Polymerase chain reaction; SIFT: Sorts intolerant from tolerant; SNP: Single nucleotide polymorphism; SNV: Single nucleotide variants; WES: Whole exome sequencing

\section{Acknowledgements}

We thank UCLA Clinical Microarray Core for their technical supports, members of Shanxi Key Laboratory of Birth Defects and Cell Regeneration for their insightful discussions. 


\section{Funding}

This research was supported by the Shanxi Province Science and Technology Research Projects (20140323001), Shanxi Provincial Natural Science Foundation (201601D102042) and Shanxi Provincial Health and Family Planning Research Projects (2015101)

\section{Availability of data and materials}

Raw data have been deposited to NCBI Bioproject website (https:// www.ncbi.nlm.nih.gov/bioproject/PRJNA352157) under the name "exome sequencing of a family of congenital contractural arachnodactyly". The SRA accession number is SRP092434 and the BioProject number is PRJNA352157.

\section{Authors' contributions}

YS, WM, QY, JX, YL, YC and CS recruited and phenotyped the patients, performed clinical evaluation and sample collection. HL performed molecular genetic experiments. $\mathrm{XL}, \mathrm{XG}, \mathrm{JX}$ and $\mathrm{CS}$ performed data analyses. CS and $\mathrm{XL}$ co-authored the draft of co-manuscript. YY, BY, HW and LQ critically revised the manuscript. All authors read and approved the final manuscript.

\section{Competing interests}

All authors declare that they have no competing interests.

\section{Consent for publication}

Written informed consent including consent for participant's details and images was obtained from each participating family member. A copy of the written consent is available for review by the Series Editor of this journal.

\section{Ethics approval and consent to participate}

All procedures in this study were approved by the Institutional Ethics Committees of Shanxi Population and Family Planning Research Institute.

Detailed written informed consent was obtained from all participants.

Received: 15 July 2016 Accepted: 24 November 2016

Published online: 03 December 2016

\section{References}

1. Epstein CJ, Graham CB, Hodgkin WE, Hecht F, Motulsky AG. Hereditary dysplasia of bone with kyphoscoliosis, contractures, and abnormally shaped ears. J Pediatr. 1968;73:379-86.

2. Grenier B, Laugier J, Soutoul J, Desbuquois G. Marfan's disease andarthrogryposis. Apropos of a case in a newborn infant. Ann Pediatr (Paris). 1969;16:182-6.

3. Beals RK, Hecht F. Congenital contractural arachnodactyly. A heritabledisorder of connective tissue. J Bone Joint Surg Am. 1971;53:987-93.

4. Lee B, Godfrey M, Vitale E, Hori H, Mattei MG, Sarfarazi M, Tsipouras P, Ramirez F, Hollister DW. Linkage of Marfan syndrome and aphenotypically related disorder to two different fibrillin genes. Nature. 1991;352:330-4.

5. Tuncbilek E, Alanay Y. Congenital contractural arachnodactyly (Beals syndrome). Orphanet J Rare Dis. 2006;1:20.

6. Frédéric MY, Monino C, Marschall C, Hamroun D, Faivre L, Jondeau G, Klein HG, Neumann L, Gautier E, Binquet C, et al. The FBN2 gene: new mutations, locus-specific database (Universal Mutation Database FBN2), and genotypephenotype correlations. Hum Mutat. 2009;30:181-90.

7. Hu W, Zhang H, Ramirez F. Developmental expression of fibrillin genes suggests heterogeneity of extracellular microfibrils. Cell Biol. 1995;129:1165-76.

8. Arteaga-Solis E, Gayraud B, Lee SY, Shum L, Sakai L, Ramirez F. Regulation of limb patterning by extracellular microfibrils. Cell Biol. 2001;154:275-81.

9. Scola RH, Werneck LC, Iwamoto FM, Ribas LC, Raskin S, Correa Neto Y. Congenital contractural arachnodactyly with neurogenic muscular atrophy: case report. Arq Neuropsiquiatr. 2001;59:259-62.

10. Jones JL, Lane JE, Logan JJ, Vanegas ME. Beals-Hecht syndrome. South Med J. 2002:95:753-5.

11. Mizuguchi T, Collod-Beroud G, Akiyama T, Abifadel M, Harada N, Morisaki T, Allard D, Varret M, Claustres M, Morisaki H, Ihara M, Kinoshita A, Yoshiura K, Junien C, Kajii T, Jondeau G, Ohta T, Kishino T, Furukawa Y, Nakamura Y, Niikawa N, Boileau C, Matsumoto N. Heterozygous TGFBR2 mutations in Marfan syndrome. Nat Genet. 2004;36:855-60.

12. Davis MR, Summers KM. Structure and function of the mammalian fibrillin gene family: implications for human connective tissue diseases. Mol Genet Metab. 2012;107:635-47.
13. Belleh S, Zhou G, Wang M, Der Kaloustian VM, Pagon RA, Godfrey M. Two novel fibrillin-2 mutations in congenital contractural arachnodactyly. Am J Med Genet. 2000;92:7-12.

14. Paulson ML, Olivier KN, Holland SM. Pulmonary Nontuberculous Mycobacterial Infection in Congenital Contractural Arachnodactyly. Int J Tuberc Lung Dis. 2012;16:561-3.

\section{Submit your next manuscript to BioMed Central and we will help you at every step:}

- We accept pre-submission inquiries

- Our selector tool helps you to find the most relevant journal

- We provide round the clock customer support

- Convenient online submission

- Thorough peer review

- Inclusion in PubMed and all major indexing services

- Maximum visibility for your research

Submit your manuscript at www.biomedcentral.com/submit
) Biomed Central 